\title{
Anomalous effect in Schumann resonance phenomena observed in Japan, possibly associated with the Chi-chi earthquake in Taiwan
}

\author{
M. Hayakawa ${ }^{1}$, K. Ohta ${ }^{2}$, A. P. Nickolaenko ${ }^{1,3}$, and Y. Ando ${ }^{1}$ \\ ${ }^{1}$ The University of Electro-Communications, Department of Electronic Engineering, 1-5-1 Chofugaoka, Chofu Tokyo \\ 182-8585, Japan \\ ${ }^{2}$ Chubu University, Department of Electronics Engineering, 1200 Matsumoto-cho Kasugai, Aichi, 487-8501, Japan \\ ${ }^{3}$ Institute of Radiophysics and Electronics, Academy of Sciences of Ukraine, Kharkov, Ukraine
}

Received: 6 December 2004 - Revised: 14 February 2005 - Accepted: 22 February 2005 - Published: 3 June 2005

\begin{abstract}
The Schumann resonance phenomenon has been monitored at Nakatsugawa (near Nagoya) in Japan since the beginning of 1999, and due to the occurance of a severe earthquake (so-called Chi-chi earthquake) on 21 September 1999 in Taiwan we have examined our Schumann resonance data at Nakatsugawa during the entire year of 1999. We have found a very anomalous effect in the Schumann resonance, possibly associated with two large land earthquakes (one is the Chi-chi earthquake and another one on 2 November 1999 (Chia-yi earthquake) with a magnitude again greater than 6.0). Conspicuous effects are observed for the larger Chi-chi earthquake, so that we summarize the characteristics for this event. The anomaly is characterized mainly by the unusual increase in amplitude of the fourth Schumann resonance mode and a significant frequency shift of its peak frequency $(\sim 1.0 \mathrm{~Hz})$ from the conventional value on the $B_{y}$ magnetic field component which is sensitive to the waves propagating in the NS meridian plane. Anomalous Schumann resonance signals appeared from about one week to a few days before the main shock. Secondly, the goniometric estimation of the arrival angle of the anomalous signal is found to coincide with the Taiwan azimuth (the unresolved dual direction indicates toward South America). Also, the pulsed signals, such as the Q-bursts, were simultaneously observed with the "carrier" frequency around the peak frequency of the fourth Schumann resonance mode. The anomaly for the second event for the Chia-yi earthquake on 2 November had much in common. But, most likely due to a small magnitude, the anomaly appears one day before and lasts until one day after the main shock, with the enhancement at the fourth Schumann resonance mode being smaller in amplitude than the case of the Chi-chi earthquake. Yet, the other characteristics, including the goniometric direction finding result, frequency shift, etc., are nearly the same. Although the emphasis of the present study is made on experimental aspects, a possible generation mechanism for this anomaly
\end{abstract}

Correspondence to: M. Hayakawa

(hayakawa@whistler.ee.uec.ac.jp) is discussed in terms of the ELF radio wave scattered by a conducting disturbance, which is likely to take place in the middle atmosphere over Taiwan. Model computations show that the South American thunderstorms (Amazon basin) play the leading role in maintaining radio signals, leading to the anomaly in the Schumann resonance.

Keywords. Ionosphere (Ionospheric disturbances) - Electromagnetics (Wave propagation) - Meteorology and atmospheric dynamics (Lightning)

\section{Introduction}

Electromagnetic phenomena associated with seismic activity have been extensively discussed, e.g. see the comprehensive monographs on these subjects by Hayakawa and Fujinawa (1994), Hayakawa (1999), and Hayakawa and Molchanov (2002). Particularly intriguing are short-term electromagnetic phenomena, which appear either as precursory signatures or as effects around the earthquake date.

Recently, a lot of evidence on the presence of such seismo-electromagnetic phenomena has accumulated. We have been using mainly two major methods of measurement for those seismo-electromagnetic phenomena. The first is the passive measurement of direct emanation of such seismogenic emissions in a wide frequency range from DC to VHF. Convincing observational evidence, especially on the seismo-ULF emissions, has been obtained for several large earthquakes (including the Spitak, Loma Prieta, and Guam earthquakes)(e.g. Hayakawa et al., 2004; Hattori, 2004; Hayakawa and Hattori, 2004). Another is the active (or radio) probing of seismo-perturbations taking place in the atmosphere and ionosphere by means of radio transmitter signals; that is, these seimo-atmospheric and ionospheric perturbations are detected in the form of propagation anomalies of transmitter signals in different frequency ranges from VLF/LF to VHF. Many anomalies in the subionospheric VLF/LF have already been observed, which enabled 

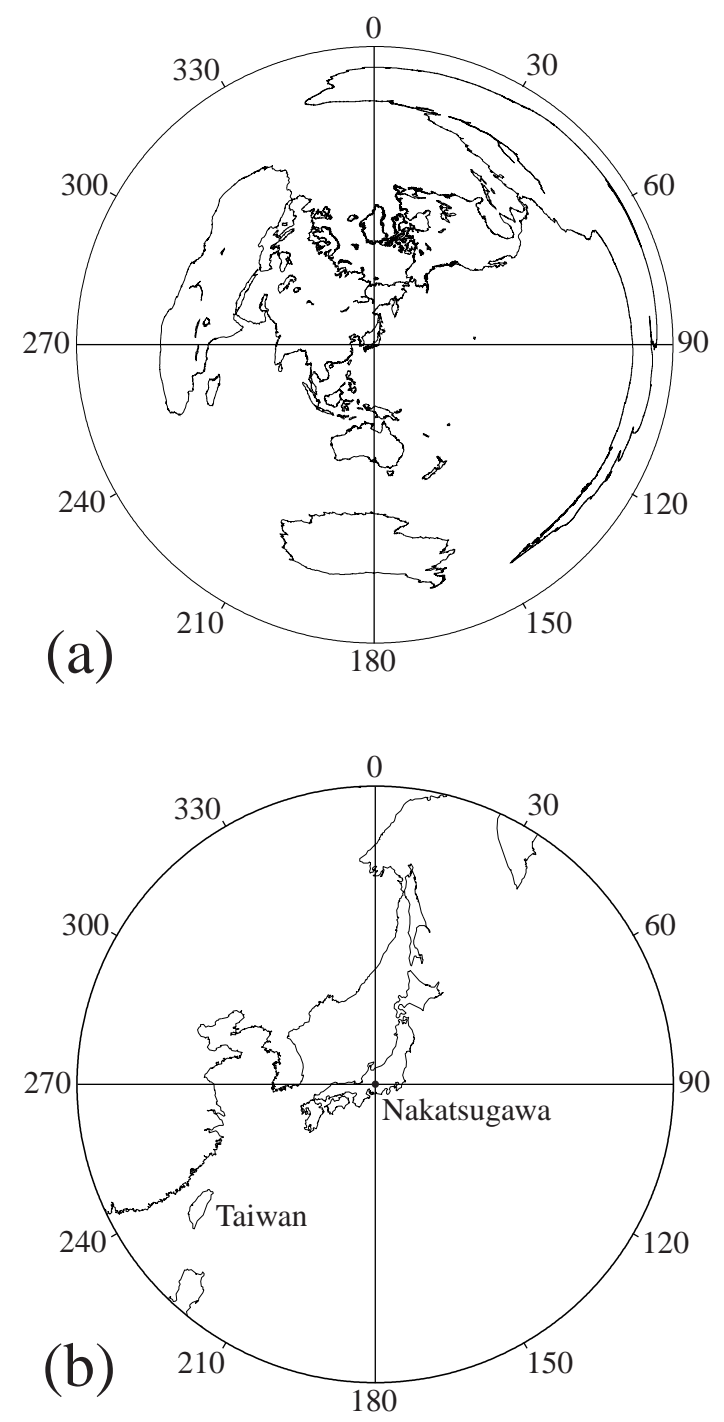

Fig. 1. (a) Relative location of our ULF/ELF observatory in Nakatsugawa and Taiwan in the form of world map with our ULF observatory located at the origin. The thunderstorm-active region in Asia is the South-east Asia, which is close to the direction of Taiwan from our ULF/ELF observatory, and South America (Amazon) is in the opposite direction. (b) The enlarged map indicating the relative location of Japan and Taiwan.

Hayakawa et al. (1996); Molchanov and Hayakawa (1998); Molchanov et al. (1998); Hayakawa et al. (2004); and Hayakawa (2004) to study the morphological characteristics of seismo-ionospheric perturbations, as well as the generation mechanism of such seismo-ionospheric perturbations in terms of the lithosphere-atmosphere-ionosphere coupling (Hayakawa et al., 2004). However, we do not think that the number of convincing seismo-electromagnetic phenomena is sufficient enough to persuade the seismologists who are still very skeptical about the presence of these seismoelectromagnetic phenomena.

In this paper we will present new records, which are completely different from those already reported in the literature.
The anomalous behaviour of the Schumann resonance signal was observed in Japan, which seems to be associated with the earthquakes in Taiwan. The Schmann resonance (SR) is a natural global electromagnetic phenomenon excited by lightning discharges mainly in the tropical region (Nickolaenko and Hayakawa, 2002). Natural oscillations are used in this paper as a radio probe of the lower ionosphere, just like the sub-ionospheric VLF/LF transmitter signals.

\section{ULF/ELF observing system in Nakatsugawa, Japan}

Our observatory is located at a very low-noise site in Nakatsugawa (geographic coordinates; $35.4^{\circ} \mathrm{N}, 137.5^{\circ} \mathrm{E}$ ), near Nagoya in Japan. The three magnetic field components $\left(B_{x}\right.$, $B_{y}$ and $B_{z}$ ) in the ULF/ELF range have been continuously measured since the beginning of 1999 at this observatory by means of three orthogonal induction coil magnetometers. The definition of $B_{x}$ is given as follows. $B_{x}$ means the geomagnetically NS component of the wave magnetic field, which can be measured by the induction magnetometer whose axis is aligned with the NS direction. So, the component of $B_{x}$ is sensitive to the waves propagating in the EW direction. Then, the $B_{y}$ component is sensitive to the waves propagating in the meridian plane. The waveform data from each channel are digitized with a sampling frequency of $100 \mathrm{~Hz}$, and are saved on a hard disk every six hours. The details of this ULF/ELF observing system has already been extensively described in Ohta et al. (2001).

\section{One year (1999) SR analysis and the Chi-chi earth- quake in Taiwan}

Figure 1 illustrates the relative location of our ULF/ELF observatory (Nakatsugawa), Taiwan (where the Chi-chi earthquake took place), and thunderstorm-active regions like Southeast Asia and South America (Amazon). We understand that the thunderstorm-active area in Southeast Asia is located approximately in the direction of Taiwan from our ULF/ELF observatory and also the American source (Amazon) is nearly in the opposite direction.

Figure 2 is the complete summary of SR behavior during the entire year of 1999 . Four groups of earthquakes in Taiwan (to be more exact, in and around Taiwan) took place with the criterion of a magnitude greater than 5.0, based on the earthquake catalogue by the Japan Meteorogical Agency. The first two earthquakes happened in May and in June, had a magnitude smaller than 6.0 and they occurred in the sea near Taiwan. No changes in the SR phenomena have been detected for these two isolated earthquakes. The third group corresponds to the Chi-chi earthquake and its aftershocks. The main shock of this Chi-chi earthquake took place with its epicenter at the geographic coordinates $\left(23.77^{\circ} \mathrm{N}, 120.98^{\circ} \mathrm{E}\right)$ and it happened at 2:47 JST (Japanese Standard Time) on 21 September 1999. The magnitude was extremely large at 7.6 and also the depth was very shallow 
$(20 \mathrm{~km})$. The circle enclosing the three successive earthquakes indicates the occurrence of the earthquakes in the land. Another anomaly in the SR phenomena was identified for the last isolated earthquake on 2 November 1999 (Chia-yi earthquake). The indication of the circles on Sch4 stands for the detection of the anomaly at the fourth SR mode, with no effect on the third SR mode (Sch3).

Below we provide you with the detailed description of the anomalous SR phenomena which are possibly associated with this Chi-chi earthquake. Lastly, we show briefly some details of the SR phenomena for the last earthquake.

\section{Anomalous behaviors in SR phenomena observed at Nakatsugawa}

The SR resonance takes place in the Earth-ionosphere cavity driven by electromagnetic radiations from lightning discharges, which are concentrated in the tropical region (Nickolaenko and Hayakawa, 2002). The fundamental frequency is $f_{1} \sim 7.8 \mathrm{~Hz}$ (the first SR mode, $\mathrm{n}=1$ ) and higher harmonics are located at $f_{2} \sim 13.9(\mathrm{n}=2), f_{3} \sim 20.0(\mathrm{n}=3), f_{4} \sim 26.0$ $(\mathrm{n}=4 \mathrm{~Hz})$, etc. Of course, we know that the SR intensity (not only fundamental, but also higher modes) depends on the source-observer distance. However, when an observer is located at middle latitude (like in Japan), the fundamental mode $\mathrm{n}=1$ is known to be usually the strongest, and the intensity is known to decrease with the mode number. We now describe the anomalous SR behaviors which are likely to be associated with the Chi-chi earthquake.

\subsection{Resonance structure}

Figure 3 shows the temporal evolution of the SR intensity, seen from the top to the bottom, the intensity at the fundamental $\left(f_{1}\right)$ and those at the harmonics $\left(f_{2}, f_{3}\right.$ and $\left.f_{3}\right)$, observed at Nakatsugawa. The magnetic component used is $B_{y}$ (measuring the magnetic field component in the EW direction, being sensitive to the waves propagating in the NS direction (nearly in the magnetic meridian plane)). A very similar tendency has been confirmed for another $B_{x}$ component, as well, so that it is not illustrated here. As is already known (Nickolaenko and Hayakawa, 2002; Sentman,1995), the resonance frequency $\left(f_{n}\right)$ is very stable (for example, the possible range in $f_{1}$ is, at maximum, $0.15 \mathrm{~Hz}$ ), but the resonance frequencies in our case are found to exhibit much more significant shifts (we will show this later), so that the bandwidth in Fig. 3 is taken to be rather large, such as fn $\pm 2.5 \mathrm{~Hz}$, and the intensity integrated over this bandwidth is plotted in Fig. 3. The unit of integrated intensity in the ordinate is the same for all resonance frequencies. The period covers only the month of September 1999, but we have to mention that the level before and after this month remains nearly the same as the background level at each resonance frequency $\left(f_{n}\right)$. It is very surprising that the integrated intensity at $f_{4}$ is extremely enhanced as compared with those at $f_{1}, f_{2}$ and $f_{3}$ during the period of 15 September to the end of September

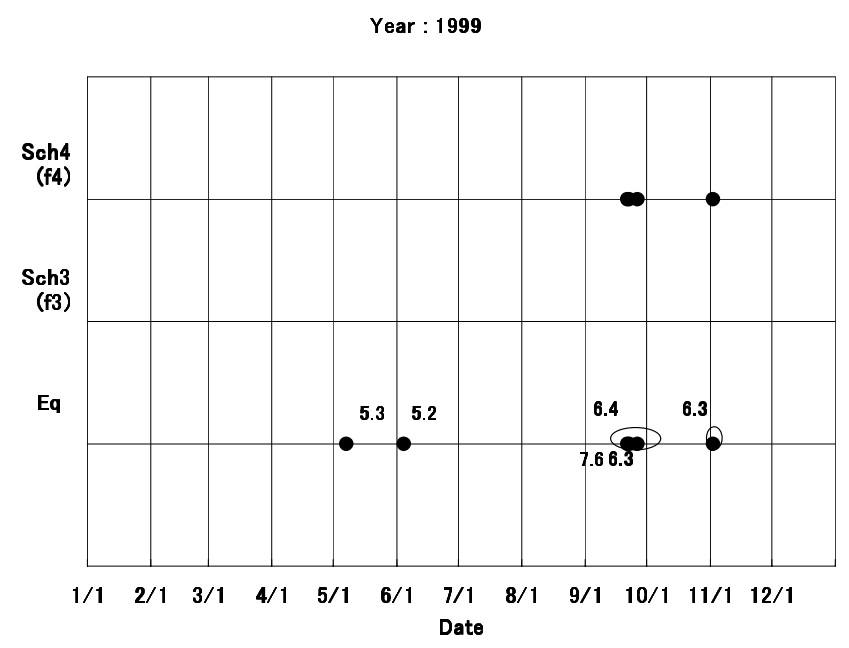

Fig. 2. One-year (1999) summary of SR observations in Nakatsugawa and anomalous SR behaviors in possible association with two large land earthquakes in Taiwan. Dots on Sch4 mean that an anomaly is taking place at the fourth SR mode, and no dots on Sch3 mean that there is no effect at the third SR mode. An earthquake with a circle indicates the land earthquake, while an earthquake without a circle means that it occurs in the sea.

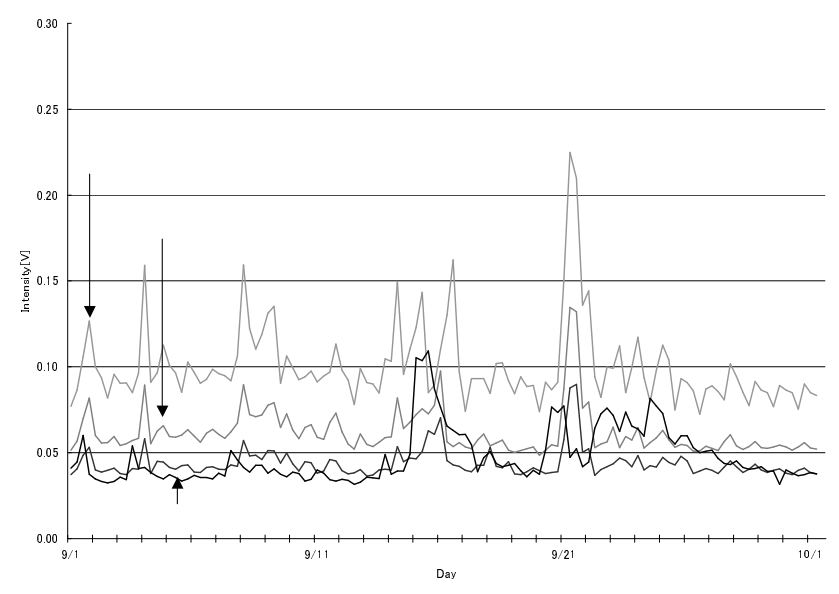

Fig. 3. Temporal evolution of the SR intensities (on $B_{y}$ ) at the fundamental $\left(f_{1}\right)$ and higher harmonics $\left(f_{2}, f_{3}\right.$ and $\left.f_{4}\right)$ in September 1999. The intensities are the values integrated over a bandwidth of $\pm 2.5 \mathrm{~Hz}$ at $8,14,20$ and $25 \mathrm{~Hz}$, respectively.

(about two weeks), which is very abnormal because the intensity at $f_{4}$ is normally much weaker than that at lower $f_{n}$ $(\mathrm{n}=1,2$, and 3) (Nickolaenko and Hayakawa, 2002). The integrated intensity at $f_{4}$ is found to show a pronounced peak starting on 15 September and decaying for a few days, with the next broad maximum with amplitude oscillation during the period of 22 to 28 September. We pay our greatest attention to $f_{4}$, but Fig. 3 shows that considerable deviations are also seen in the amplitudes of other lower-order modes.

Figure 4 illustrates the above temporal evolution for the same $B_{y}$ component in a different form as the dynamic spectrum. The period is again the same, September 1999, and 


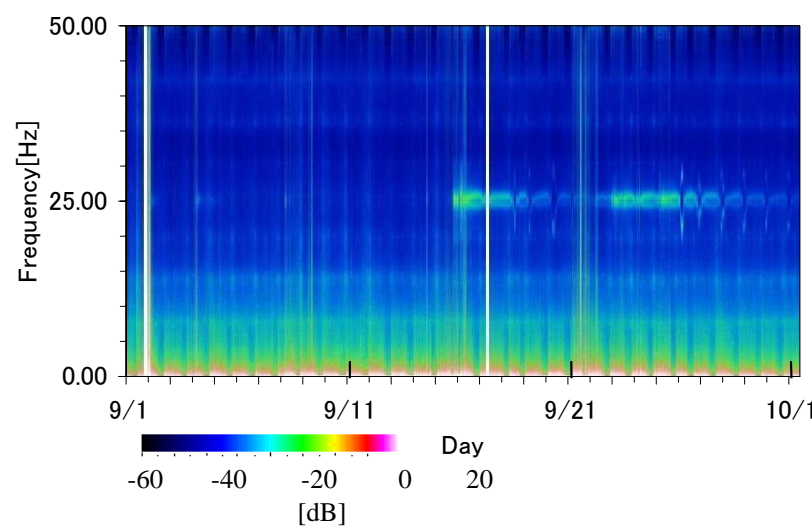

Fig. 4. Temporal evolution of dynamic spectrum of the SR intensity in September 1999. The intensity is indicated in color.
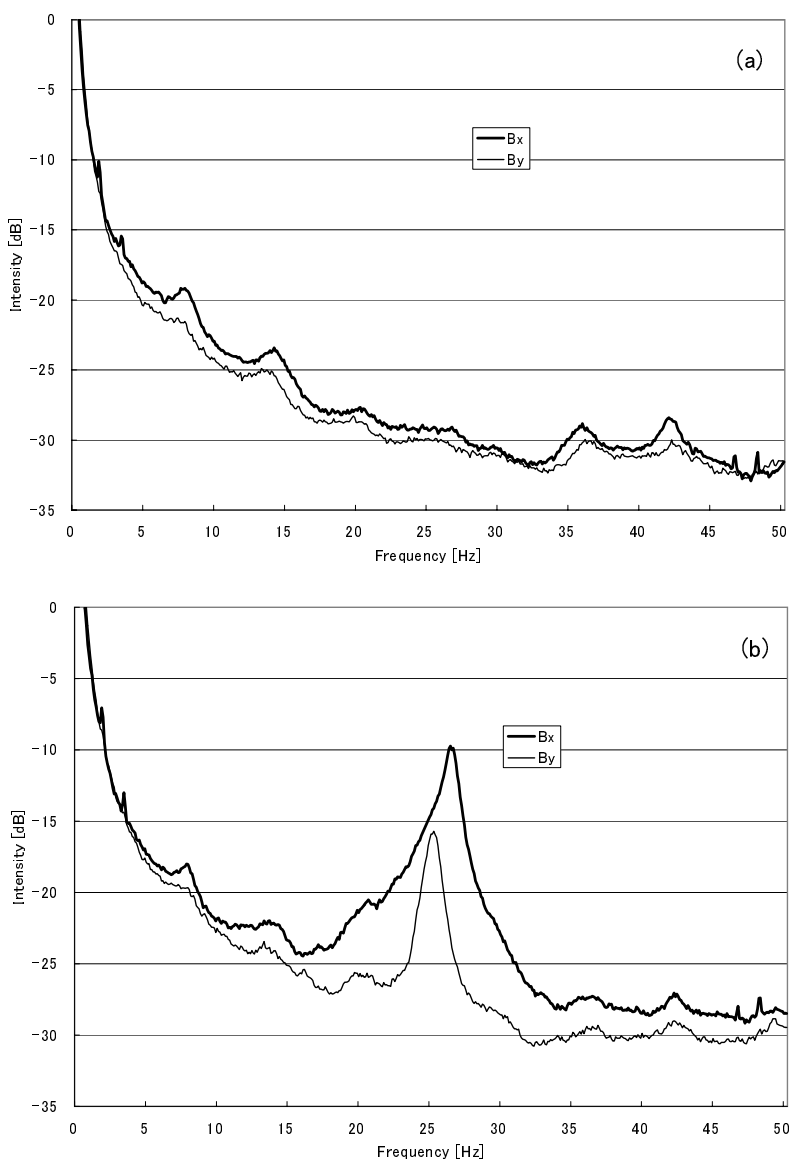

Fig. 5. Frequency spectra of SR on 10 September (normal condition) (a) and on 16 September (abnormal condition) (b). The two magnetic components $\left(B_{x}\right.$ and $\left.B_{y}\right)$ are shown.

the frequency is in a range up to $50 \mathrm{~Hz}$. The wave intensity is indicated in the given color code. As seen from Fig. 4, the intensity of higher harmonics $\left(f_{4}\right)$ is extremely enhanced from 15 September to the end of September. Figure 5 illustrates the frequency spectra on two particular days; 10 September (normal condition) $(\mathrm{LT}=6 \mathrm{~h}-12 \mathrm{~h})$ and on 15 September (ab-

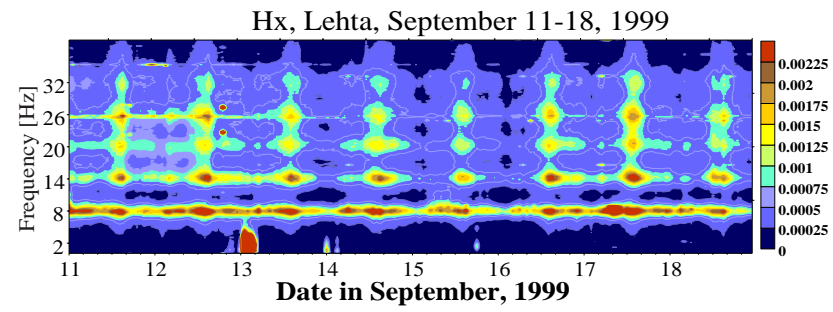

Hy, Lehta, September 11-18, 1999

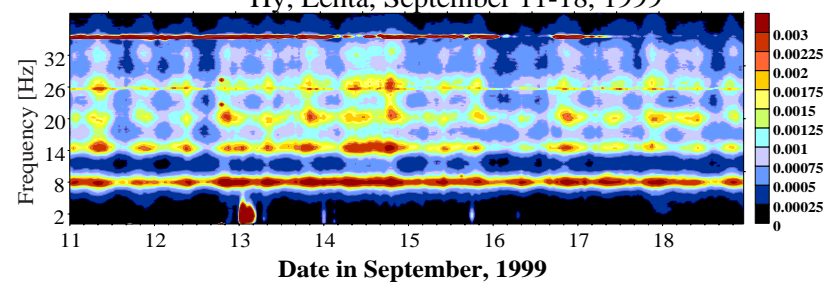

Fig. 6. Corresponding dynamic spectra of the SR at the Lekhta station (as a reference station) during the same September 1999.

normal condition) (LT=6 h-12 h), as observed by the $B_{x}$ and $B_{y}$ components. One notices easily the enhancement at $f_{4}$, with a significant frequency shift on both components. For comparison, we plot in Fig. 6 the corresponding SR phenomena observed at Lekhta (geographic coordinates; $64.43^{\circ} \mathrm{N}$, $33.97^{\circ} \mathrm{E}$ ), Karelia, Russia during the same period. We cannot find any significant anomalous behavior, especially in the fourth mode in Lekhta. The intensity is definitely decreasing with increasing mode number and also the resonance frequencies $\left(f_{n}\right)$ are found to be the conventional values. There is a narrow line just below $26 \mathrm{~Hz}$, but it is apparently an artificial noise.

4.2 Shift in resonance frequencies, asymmetry in the resonance frequency for $B_{x}$ and $B_{y}$ components and resonance intensity

We describe in more detail the anomalies in the SR. As is seen in Figs. 3, 4 and 5, the fourth harmonic $\left(f_{n}\right)$ is extremely enhanced in the dynamic spectra. We have measured the frequency of the fourth harmonic $\left(f_{4}\right)$ on the two horizontal magnetic field components $\left(B_{x}\right.$ and $\left.B_{y}\right)$. As mentioned before, one file has a duration of $6 \mathrm{~h}$, so that we have integrated the intensity over $6 \mathrm{~h}$ only to find the frequency of maximum intensity. Figure 7 illustrates this temporal variation of the frequency $\left(f_{4}\right)$ on the two magnetic components $\left(B_{y}\right.$ and $B_{x}$ ) during the anomalous period of 15 September to the end of September. The abscissa of Fig. 7 indicates the date (e.g. 15 means 15 September) and there are four divisions in one day with file numbers $0,1,2$ and 3 (e.g. 0 means $0-6 \mathrm{~h} \mathrm{LT}, 1$ means $6-12 \mathrm{~h}$ LT and so on). We continue cover the same September 1999. We have to comment on the frequency resolution in estimating Fig. 7. We use the data length of $1,024 \times 10 \mathrm{~ms}(\sim 10 \mathrm{~s})$, so that the frequency resolution is $0.098 \mathrm{~Hz}$ and the time resolution is $10 \mathrm{~s}$. Figure 7 indicates that the central resonance frequency $\left(f_{4}\right)$ is changing from $25.4 \mathrm{~Hz}$ on 15 September to $25.2 \mathrm{~Hz}$ on 21 
September for the magnetic component $B_{y}$, while the corresponding resonance frequency $\left(f_{4}\right)$ is found to be much more stable than the $B_{y}$ behavior in such a way that the resonance frequency $\left(f_{4}\right)$ is found to be just around $26.3 \mathrm{~Hz}$. As compared with the previous experimental findings, see Nickolaenko and Hayakawa (2002) and recent measurements (Price and Melnikov, 2004), the resonance frequency for the $B_{y}$ component is found to be significantly lower than the conventional value by $0.6-0.8 \mathrm{~Hz}$. However, the resonance frequency for the $B_{x}$ component is seen to be slightly (but significantly) different from the conventional value by $+0.3 \mathrm{~Hz}$ (+ means higher than the conventional value).

The $B_{y}$ component in Fig. 7 is reflecting the wave activity propagating in the NS direction (nearly in the magnetic meridian plane), that is, this $B_{y}$ component seems to be very sensitive to the nearest Asian thunderstorm activity. It is obvious that the diurnal variation of the resonance frequency for the $B_{y}$ component exhibits a very clear diurnal variation in such a way that we expect a minimum for the file numbers 1 or 2 ; that $\mathrm{LT}=6-12 \mathrm{~h}$ or $12 \mathrm{~h}-18 \mathrm{~h}$ (corresponding to the Asian thunderstorm activity), and a maximum around midnight in LT (larger source-observer distance (for American and African sources) by considering that the resonance frequency is closely related to the source-observer distance for the three sources (Nickolaenko and Hayakawa, 2002). This experimental diurnal variation is likely to be consistent with the theoretical expectation as given in Nickolaenko and Hayakawa (2002). Here we have to comment on the diurnal variation for another $B_{x}$ component (which is sensitive to the waves propagating mainly in the EW direction). A close inspection of Fig. 7 indicates that the $B_{x}$ component in Fig. 7 seems to show the similar diurnal variation as for the $B_{y}$ component, but much less pronounced than for the $B_{y}$ components. Altogether these suggest that the abnormal behaviors, as presented in this paper, are fundamentally supposed to be closely related to the thunderstorm activity in the tropical region.

How about the diurnal variation of the intensity at $f_{4}$ for both magnetic components? Although not shown as a figure, we have found that the diurnal variation of the intensity is not so significant for both magnetic components $\left(B_{x}\right.$ and $\left.B_{y}\right)$. This seems to be consistent with the previous experimental findings (Nickolaenko and Hayakawa, 2002).

\subsection{Direction finding results}

Because of the significant difference in the central frequencies of SR at $f_{4}$ on the two magnetic components, the goniometer direction finding is performed for each component $\left(B_{x}\right.$ or $\left.B_{y}\right)$. When we perform the goniometric direction finding to the SR phenomena anomaly observed for the $B_{y}$ component, we first estimate the maximum intensity on the $B_{y}$ component with the previously-mentioned frequency every $10 \mathrm{~s}$ and we perform the goniometric direction finding as is given in Hayakawa (1995) at those frequencies in a range from 25.1 to $26.0 \mathrm{~Hz}$ by using the radio of $B_{x}$ and $B_{y}$ components. Figure 8a illustrates the occurrence histograms of the

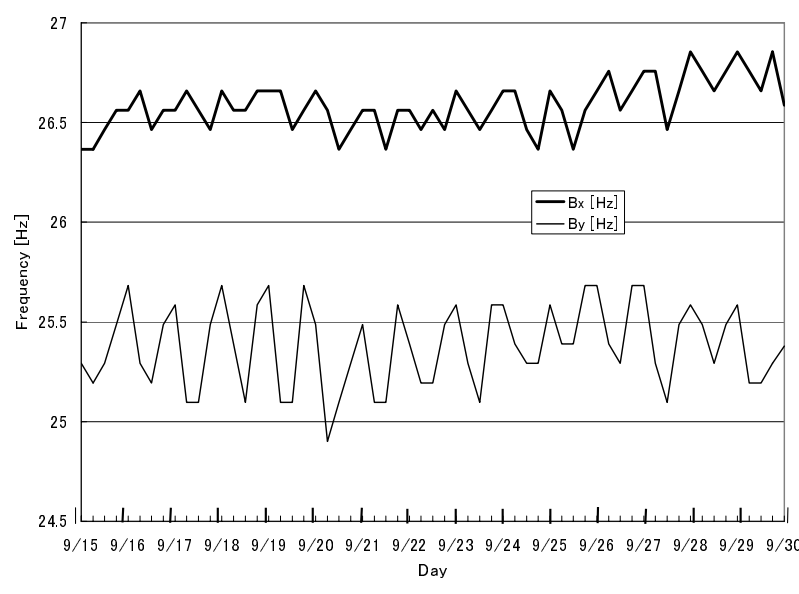

Fig. 7. Detailed temporal evolutions of the fourth resonance frequency $(f 4)$ on the two magnetic antennas $\left(B_{x}\right.$ and $\left.B_{y}\right)$ during the same September.

phase difference between the components $B_{x}$ and $B_{y}$ (with $B_{y}$ as the reference, $B_{y}-B_{x}$ ), indicating that the occurrence number is well peaked around $0^{\circ}$ (suggesting the linear polarization). This phase information enables us to adopt the principle of the goniometer to estimate the azimuthal direction for the waves. Figure $8 \mathrm{~b}$ is the result, which means that the waves are coming from the azimuth of $215^{\circ}$ with a rather wide distribution. This central direction is close to the direction of Taiwan $\left(235^{\circ}\right)$ and that of the Asian thunderstorm source region and the American source (Amazon), as is seen from Fig. 1.

Figures $9 \mathrm{a}$ and $\mathrm{b}$ are the corresponding direction finding results for the $B_{x}$ component. Figure $9 \mathrm{a}$ is the occurrence histogram of the phase difference between $B_{x}$ and $B_{y}$ (with $B_{x}$ as the reference, $B_{x}-B_{y}$ ), which indicates a broad distribution with a peak around $\sim 60^{\circ}$. Even in this situation we perform the goniometric finding, and the result is illustrated in Fig. 9b, which indicates $\sim 90^{\circ}$ (measured eastward from the north). Probably there must be a significant polarization error in the estimation of azimuth in Fig. 9b (as is suggested in Hayakawa (1995)) because of a significant departure of the phase difference from $0^{\circ}$. However, the source in Fig. $9 \mathrm{~b}$ might be American (Amazon).

\subsection{Associated ELF transients and ULF emissons}

One notices the presence of ELF transients (Q bursts) in Fig. 4, which indicates the pulsive noises (or pulsive spectra) superimposed on the enhanced SR at $f_{4}$ from 15 September to the end of September. The occurrence of simultaneous ELF transients is observed during the same period of enhanced SR intensity at $f_{4}$ from 15 September to the end of September. Normal Q-bursts are known to have the main frequency of oscillations just around strongest fundamental resonance frequency (Ogawa et al., 1967; Nickolaenko and Hayakawa, 2002), which is the simple manifestation of the most preferred Earth-ionosphere propagation condition. The 

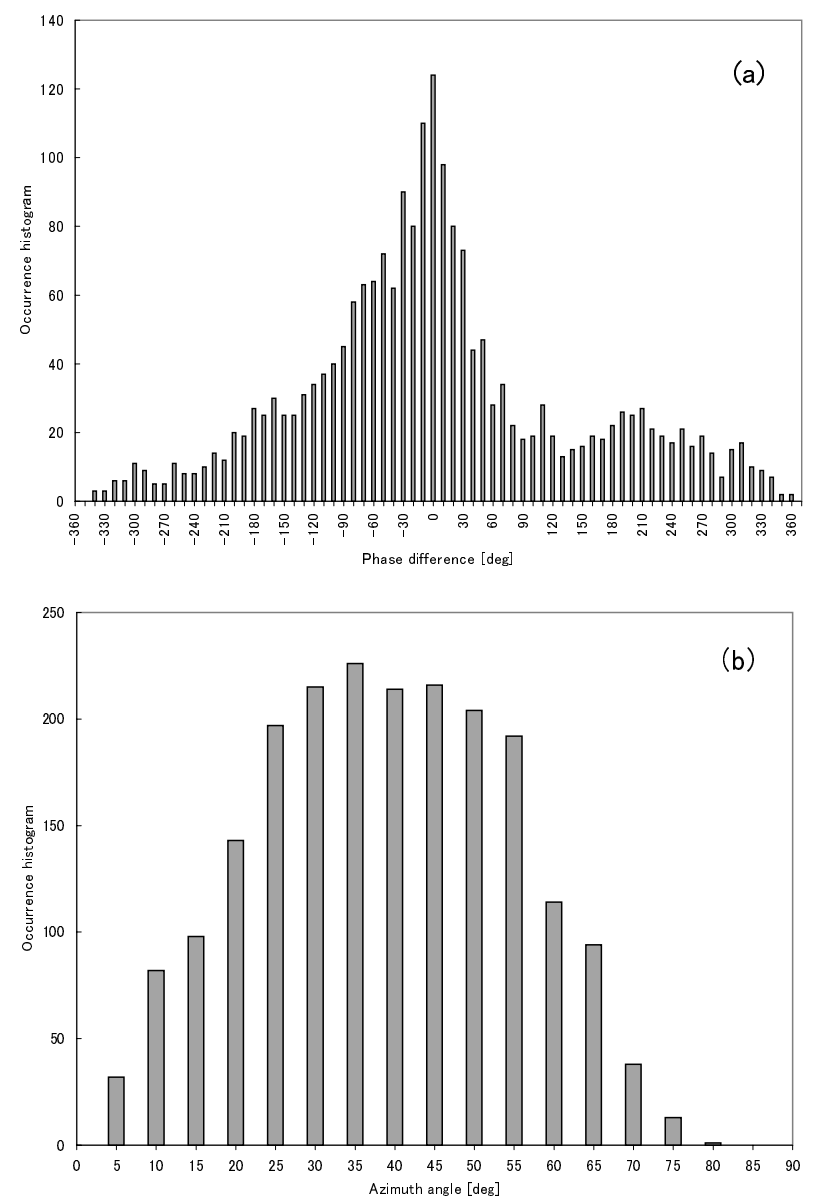

Fig. 8. (a) Occurrence histogram of the phase difference in a frequency range of $25.1 \sim 26.0 \mathrm{~Hz}$ (with $B_{y}$ component as the reference) and (b) the occurrence histogram of the obtained arrival direction (measured from north to east).

main frequency of our ELF transients in Fig. 4 is clearly not at $f_{1}$, but just around the $f_{4}$. Again, we have performed the goniometric direction finding for those ELF transients, and we have found that the azimuthal direction of those ELF transients is mainly distributed around $235^{\circ}$ (which is the direction toward Taiwan). Figure 4 indicates, as well, the presence of the associated ULF transients which have already been extensively discussed for this Chi-chi earthquake by Ohta et al. (2001).

\subsection{Repeatability}

Now we want to add one more important point for this anomalous SR phenomena; repeatability of this anomaly. Although Fig. 3 indicates that the intensity variation at $\sim f_{4}$ with a peak on 15 September and with decaying for a few days might be associated with the Chi-chi earthquake, we find an additional broad peak on 22 September to 26 September (a little complicated, but seeming to be one event) in this figure. An aftershock of the Chi-chi earthquake is known to have taken place nearly at the same place at 8:53 JST on 26
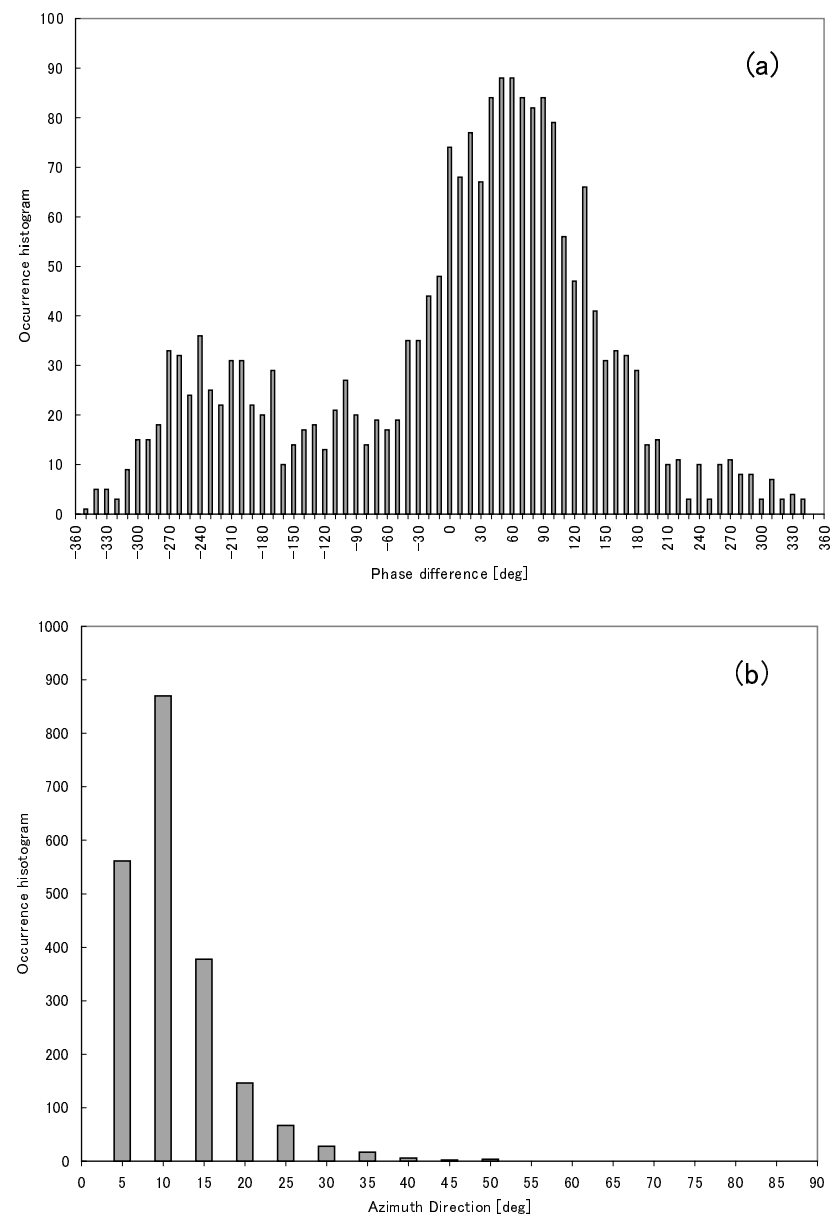

Fig. 9. The same as Fig. 8, expect for the $B_{x}$ component in the frequency range of $26.2-26.7 \mathrm{~Hz}$.

September, which may suggest the peak on 22-26 September is closely associated with this aftershock. Of course, there may be a possibility that this broad peak might be the aftereffect of the Chi-chi earthquake. We cannot say which interpretation may be plausible. However, it seems more likely by the description of the anomalous SR event for another large earthquake in Taiwan (as already shown in Fig. 2) that the first peak may be related to the Chi-chi earthquake and the second broad peak, its aftershock.

Here we have to comment on the anomalous SR phenomena in possible association with the earthquake on 2 November 1999 (Chia-yi earthquake), as is already shown in Fig. 2. The most important and exciting point for this earthquake is that the anomaly is taking place again in the fourth resonance mode. Figure 10 illustrates the dynamic spectra one day before the earthquake (i.e. 1 November 1999, 6:00-18:00 JST). We summarize the SR anomaly which is probably associated with this earthquake. The enhancement of the SR intensity at the fourth resonance mode is found to occur one day before the earthquake and it lasts for two days. The highest intensity was observed about $14 \mathrm{~h}$ before the earthquake. But the intensity and duration of this anomaly is likely to be much 
smaller than those for the Chi-chi earthquake, which might be related to its much smaller magnitude. Other characteristics (goniometric direction finding result, frequency shift, etc.) are found to be nearly the same as those for the Chi-chi earthquake.

\section{Summary of the anomalous SR behavior, possible as- sociation with the Chi-chi earthquake and discussion on the generation mechanism}

We can summarize the anomalous SR behaviors observed at Nakatsugawa prior to two large earthquakes which took place in Taiwan in September and November, 1999.

1. Unlike the usual situation of SR, the intensity of the fourth mode $\left(\sim f_{4}\right)$ for the Chi-chi earthquake is enhanced at Nakatsugawa during the period from 15 September to the end of September 1999. Also, there is an enormous asymmetry in the resonance frequency $\left(f_{4}\right)$ for both magnetic components, $B_{x}$ and $B_{y}$. The $B_{y}$ component (sensitive to the wave coming from the NS direction) and $B_{x}$ as well exhibit the enhancement at $f_{4}$, but the exact resonance frequency for the $B_{x}$ component is about $1 \mathrm{~Hz}$ lower than the conventional value of $26.0 \mathrm{~Hz}$, and the corresponding value for another magnetic component $\left(B_{x}\right)$ is slightly higher than the conventional value by $+0.3 \mathrm{~Hz}$. This kind of SR anomaly was not seen in Lekhta, where the SR characteristics are exactly in the normal condition.

2. The arrival azimuth for the SR wave around $25.2 \mathrm{~Hz}$ mainly for the $B_{y}$ component is found to be $\sim 250^{\circ}$, very close to the direction of Taiwan, while that for the SR wave at $26 \mathrm{~Hz}$ mainly for the $B_{x}$ component, is found to be directed forward South America (or Amazon).

3. Another important finding is the simultaneous occurrence of ELF transients, but their frequency is just around $f_{4}$, very similar to the Q-burst as observed by Ogawa et al. (1967). The direction finding for those ELF transients indicates that their azimuth is exactly in the direction of Taiwan.

This kind of rare case was also observed for another large (Chia-yi) earthquake in Taiwan (in November 1999) with characteristics similar to the Chi-chi case.

\section{Discussion and interpretation}

First, we have to discuss whether this kind of unusual behavior in SR is a natural phenomenon or not, and whether this anomaly is related to the Chi-chi earthquake. Assuming that this is a kind of artificial noise (like train noise), the emission line must then be much narrower than our event. The Q factor of the fourth resonance $\left(f_{4}\right)$ in our case is of the order of $\sim 10$, which is a further indication of natural phenomenon, though this value is nearly twice the conventional SR value
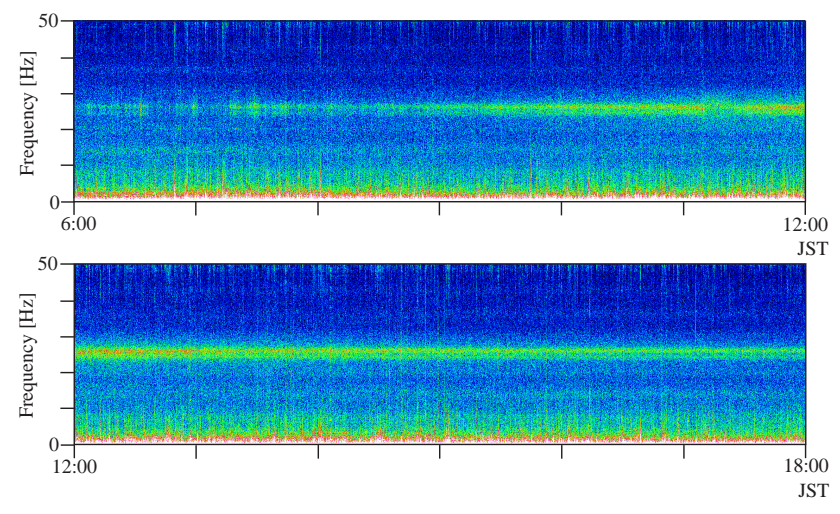

Fig. 10. Dynamic spectra of the SR at Nakatsugawa one day before the earthquake (2 November 1999) (i.e. 1 November 1999, JST=6:00-18:00), in which one notices an anomaly in the fourth resonance mode in possible association with the earthquake on 2 November 1999.

as given in Nickolaenko and Hayakawa (2002). Additional observational facts in support of this natural noise effect are (1) the $B_{y}$ component (also on the $B_{x}$ component, but not so prominent) exhibits a typical diurnal variation in resonance frequency at $\sim f_{4}$ and (2) the direction finding for the emission at $\sim f_{4}$ for the $B_{y}$ component shows that it is directed incidentally toward the direction of Taiwan and also the direction of America. The first observational fact is strongly indicating that the source of this anomaly is likely to be the global thunderstorm-active region in Southeast Asia and America, which is observationally supported by the 2nd direction finding result. Therefore, we can conclude that this anomaly is a real typical SR phenomenon with anomalous behaviors.

The next problem is whether this anomaly in SR phenomena is really associated with the Chi-chi earthquake or not. The first point is that this anomaly in SR is taking place before the Chi-chi earthquake, which is not so convincing as a precursory effect of an earthquake. Of course, it may be possible for us to think it just as a coincidence. However, we have shown the repeatability of this kind of SR anomaly again for another big earthquake in Taiwan. This would lend us some more convincing support to our supposition that those anomalies are the consequence of the big earthquakes. Some other big earthquakes in Japan have produced similar behaviour (of course, not exactly the same, but the higher harmonics had some abnormal behaviors), which will be our next paper, no effect was completely confirmed at Lekhta (Karelia, Russia). Further, smaller earthquakes with magnitudes less than 6.0 had no effect on the SR, as shown in Fig. 2, which may be an additional support that the anomalous SR phenomena might be earthquake-related.

ELF transients or bursts have been observed during the period of 15 September to the end of September when anomalous SR phenomena were detected. Also, we have found a lot of ULF/ELF (mainly in the frequency range less than $10 \mathrm{~Hz}$ ) in association with the ELF transients, which is clearly seen in Fig. 4. The main frequency of ELF transients or Q bursts 


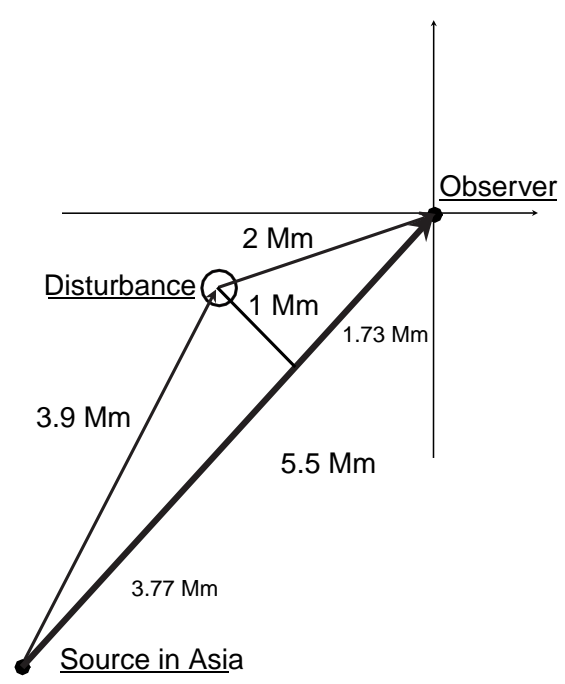

(a) ASIA (Indonesia)

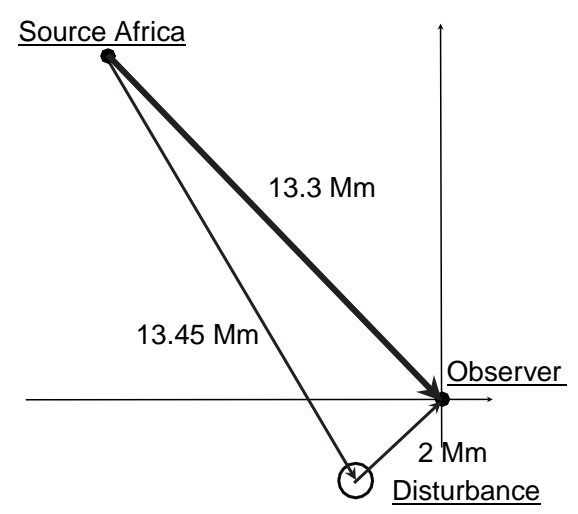

(b) AFRICA

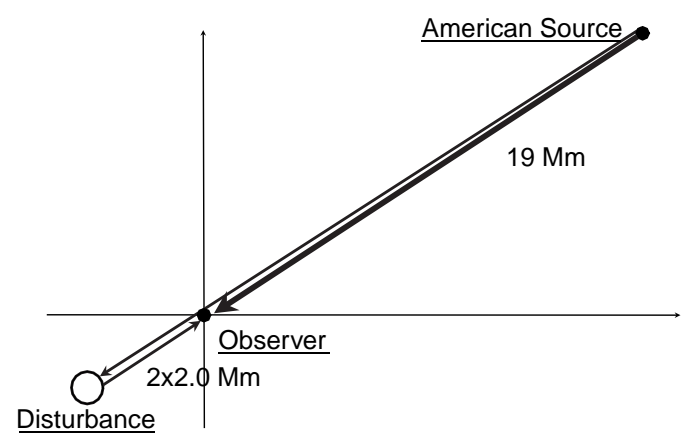

(c) AMERICA

Fig. 11. Three thunderstorm regions (a) Asia (Indonesia), (b) Africa and (c) America (Amazon) and the configurations of the direct path and that scattered at Taiwan are given for each source. is known to be the fundamental frequency of SR (Ogawa et al., 1967; Nickolaemko and Hayakawa, 2002). The generation of ULF emission has already been confirmed by Ohta et al. (2001) to have the goniometric direction toward Taiwan. This might indicate an additional noise source in Taiwan, which may be the precursory to the Chi-chi earthquake.

How to explain various characteristics of the anomaly described in this paper, is poorly understood, so that we propose one possible hypothesis. The two important points of the SR anomaly and (1) the fourth resonance is extremely enhanced for the $B_{y}$ component and (2) a significant shift of the order of a little less than $1.0 \mathrm{~Hz}$ for the $B_{y}$ component. When taking into account different properties, the source for the SR anomaly is highly likely to be the thunderstorm in the Southeast Asia or in the South America (Amazon region). We initially assume that the ionosphere (or in the atmosphere) above the epicenter of the Chi-chi earthquake is perturbed, acting as a scatterer (or a reflector). We then think about three possible thunderstorm centers in Asia, Africa and America. The configurations for a direct path and the path scattered at Taiwan for those three sources are summarized in Fig. 11. In the case of the Asian source (Fig. 11a), the source-observer distance is $5.5 \mathrm{Mm}$, and the path difference between this direct path and the path scattered at Taiwan is about $0.4 \mathrm{Mm}$. The maximum effect (strongest wave interference effect) is expected when this path difference is equal to $\lambda / 2$ ( $\lambda$ : wavelength). Then we obtain $\lambda=0.8 \mathrm{Mm}$. Since $\lambda=40 \mathrm{Mm}$ at the first SR mode, the above wavelength is found to correspond to the $40 / 0.8=50$ mode number, or to the frequency of approximately $300 \mathrm{~Hz}$. So, Asia is the poorest candidate for strong wave interference at $\sim f_{4}$.

We move on to the next source of Africa (Fig. 11b); source-observer distance $=13 \mathrm{Mm}$, and the path difference between the direct path and that scattered at Taiwan is $2.15 \mathrm{Mm}$. The maximum effect is reached when the path difference is equal to $\lambda / 2$ and hence $\lambda=4 \mathrm{Mm}$. This means that the above wavelength corresponds to the 40/4=10 mode number, or to the frequency of about $60 \mathrm{~Hz}$. So, Africa is found to be not good, but not so bad either. The last source of America is considered (Fig. 11c); the source-observer distance $=19 \mathrm{Mm}$ and the path difference between the direct path and the path scattered at (or reflected from) Taiwan $=4 \mathrm{Mm}$. We can expect the maximum wave interference effect when this path difference is $\lambda / 2$, so that $\lambda=8 \mathrm{Mm}$. This wavelength is found to correspond to the $40 / 8=5$ mode number, or to the frequency of approximately $32 \mathrm{~Hz}$. In this sense, the American source (Amazon) is found to be the most reasonable candidate for the source position. Fortunately, the direction (azimuth) of Taiwan is nearly in the same direction to the Amazon, so that we can think of a very simple situation. The observer receives both the direct signal from the American source and the signal reflected from the ionospheric perturbation located at Taiwan, as is seen is Fig. 11c.

We try to give some computations for this situation. We treat the scattering of the primary SR wave by a localized ionospheric disturbance. For simplicity, we consider the vertical electric field component because its orientation is 
Table 1. Coordinates, distances to the observer, and geometrical parameters of the model.

\begin{tabular}{lrrr}
\hline $\begin{array}{l}\text { Thunderstorm } \\
\text { center }\end{array}$ & Asia & Africa & America \\
\hline Coordinates & $0^{\circ} \mathrm{N}, 120^{\circ} \mathrm{E}$ & $5^{\circ} \mathrm{N}, 10^{\circ} \mathrm{E}$ & $0^{\circ} \mathrm{N}, 50^{\circ} \mathrm{W}$ \\
$\begin{array}{l}\text { Source-observer } \\
\text { distance (in Mm) }\end{array}$ & 2.7 & 12.0 & 17.2 \\
$\cos \left(\alpha_{i}\right)$ & 0.95 & 0.96 & 0.89 \\
$M_{i}$ & 0.76 & 0.52 & -0.43 \\
\hline
\end{tabular}

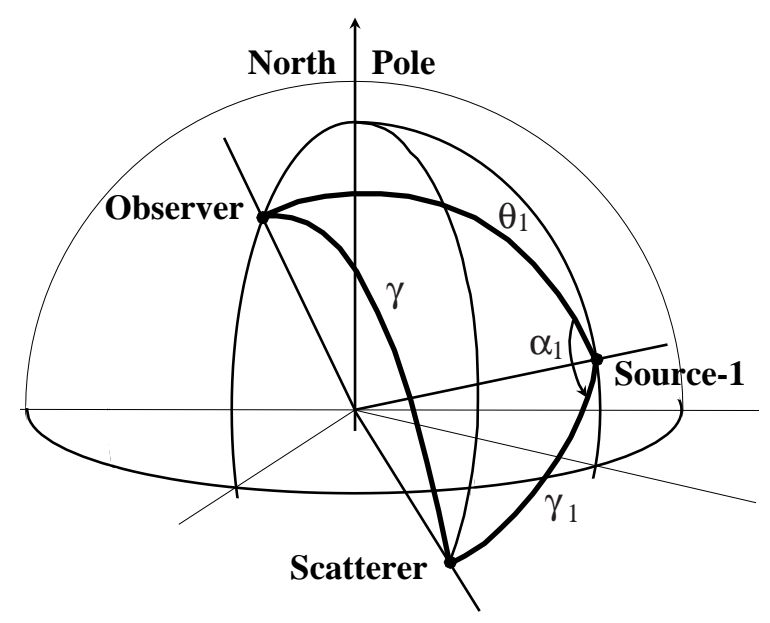

Fig. 12. Configuration of our scattering problem.

independent of the mutual positions of the field source, observer and disturbance. The vertical electric field is a sum of the direct (primary) $\left(E_{1}\right)$ and scattered $\left(E_{2}\right)$ waves:

$E=E_{1}+E_{2}$.

The direct (or primary) wave is defined simply by the formula (Eqs. (4.16) and (4.18) in Nickolaenko and Hayakawa (2002).

$E_{1}(\omega)=\frac{M(\omega)}{4 h a^{2} \varepsilon} \frac{i v(v+1)}{\omega} \frac{P_{v}\left[\cos \left(\pi-\theta_{i}\right)\right]}{\sin \pi v}$.

Here $\omega$ is the wave angular frequency, $M(\omega)$ is the source current moment, $v(\omega)$ is the propagation constant depending on the frequency, $h$, the ionospheric height, $a$, the Earth's radius and the $\varepsilon$, dielectric constant of free space. Here we use the linear frequency dependence, $v(f)=(f-2) / 6-i \cdot f / 70$ (Nickolaenko and Hayakawa, 2002). $P_{v}(\cos \theta)$ is the Legendre function, and the angular distance $\theta_{i}$ acquires the values $\theta_{1}, \theta_{2}$, or $\theta_{3}$, corresponding to the sources placed in Asia, Africa and America in Fig. 11. Figure 12 shows the geometry of this scattering problem. It is convenient to introduce individual fractional contributions from the ionospheric disturbance into the field arriving from different sources:

$B=\frac{E_{2}}{E_{1}}=\frac{\int \sin \theta d \theta d \varphi Q_{i} \delta C_{v}^{2}}{4 \sin \pi v P_{\nu}\left[\cos \left(\pi-\theta_{i}\right)\right]}$, where

$Q_{i}=v(v+1) P_{v}\left[\cos \left(\pi-\gamma_{i}\right)\right] P_{v}[\cos (\pi-\gamma)]$

$-M P_{v}^{1}\left[\cos \left(\pi-\gamma_{i}\right)\right] P_{v}^{1}[\cos (\pi-\gamma)]$

where $P_{v}^{1}(\cos \theta)$ is the associated Legendre function, $\gamma$ is the angular distance from the observer to disturbance, $\gamma_{i}$ is the distance from the disturbance to the given source in Asia, Africa, or America. The geometrical parameter of differentiation is found from the following relation, $\quad M=\frac{\partial \gamma_{i}}{\partial \gamma}=\frac{\sin \theta_{i} \cos \gamma_{i} \cdot \cos \alpha_{i}-\sin \gamma_{i} \cos \theta_{i}}{\sin \gamma} \quad$ (see Nickolaenko and Hayakawa, 2002). We suppose that the ionospheric modification locally changes the propagation parameter $\delta C_{v}^{2}=\delta\left(1-\frac{v(v+1)}{(k a)^{2}}\right)=-i \frac{\delta Z}{k h}(k$, propagation constant of free space and $\delta \mathrm{Z}$, the change in surface impedance) (Nickolaenko, 1994), and this latter has a symmetric "Gaussian" angular distribution:

$\delta C_{v}^{2}=\Delta C_{v}^{2} \exp \left(\frac{\cos \beta-1}{d^{2}}\right)$.

Here $\Delta C_{v}^{2}$ is the maximum disturbance, $\beta$ is the angular distance from the center of ionospheric modification to the point of integration, and $d$ is the characteristic size of the modification. One readily obtains the following formula after integrating Eq. (3) in the above case of a compact, localized non-uniformity:

$B=\frac{E_{2}}{E_{1}}=\frac{\pi d^{2}}{2} \frac{\Delta C_{v}^{2} \cdot Q_{i}}{\sin (\pi \nu) \quad P_{v}\left[\cos \left(\pi-\theta_{i}\right)\right]}$.

We use the following model in our computations. The observer is located at Nakatsugawa, Japan $\left(35.45^{\circ} \mathrm{N}\right.$ and $\left.137.3^{\circ} \mathrm{E}\right)$. The disturbance is placed over Taiwan whose geographic coordinates are $24^{\circ} \mathrm{N}$ and $122^{\circ} \mathrm{E}$, and its characteristic size $d$ corresponds to $1000 \mathrm{~km}(d=\pi / 20)$. We suppose that one of three global thunderstorm centers drives the SR signal. The coordinates and relevant parameters of geometry are listed in Table 1. In our computations at lower ELF SR frequencies for the possible earthquake disturbance we formally increased the disturbance of the complex cosine by a factor of $(40 / \pi)$ in our comparison, by using the value used at the frequency of $75 \mathrm{~Hz}$ (Pappert, 1981): $\Delta C_{v}^{2}=\frac{40}{\pi} R_{a} \cdot C_{v}^{2}$. The factor $R_{a}$ was introduced for interpretations of the nighttime disturbances observed experimentally in the ELF signal from the US Navy transmitter (Wisconsin Test Facility), and its value is $R_{a}=0.13617-i \cdot 10.6949$ (see Nickolaenko and Hayakawa, 2002). We must keep in mind that the amplitude of the disturbance does not play a key role now. We try to establish whether a localized disturbance over Taiwan might produce an effect similar to that observed experimentally. We also hope to see the impact of the source position on the resulting field modifications. The particular value of disturbance and relevant type of ionospheric modification (whether this is a change in the effective height, or in the effective surface impedance) might be found later in a detailed comparison of special computational and measured data. It is also 

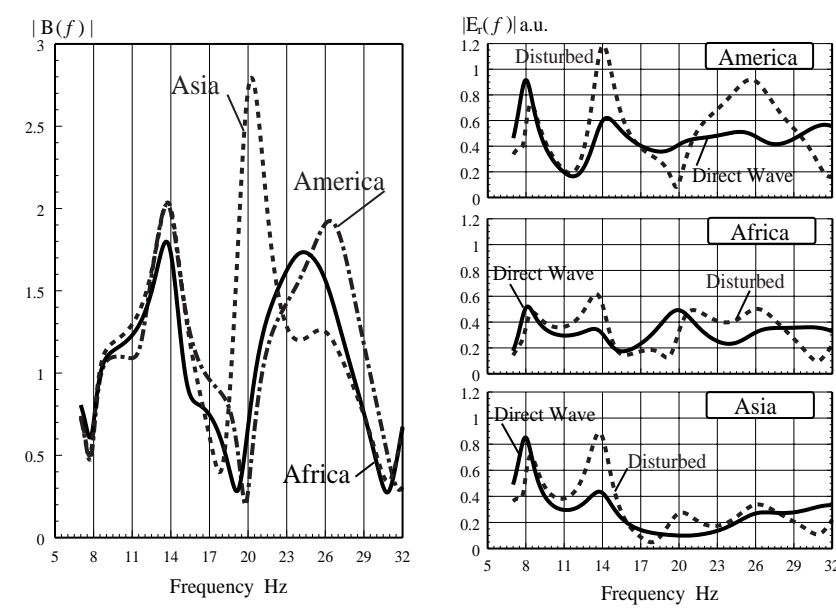

Fig. 13. Left panel shows the computational results on the frequency dependence for three thunderstorm centers. The right panel indicates the frequency dependences of the vertical electric field expected at our observatory for three sources (from the top to the bottom; America, Africa and Asia). A thin line refers to the direct wave without the effect of ionospheric perturbation, while a thick line, the corresponding result for the disturbed case (with the ionospheric perturbation over Taiwan).

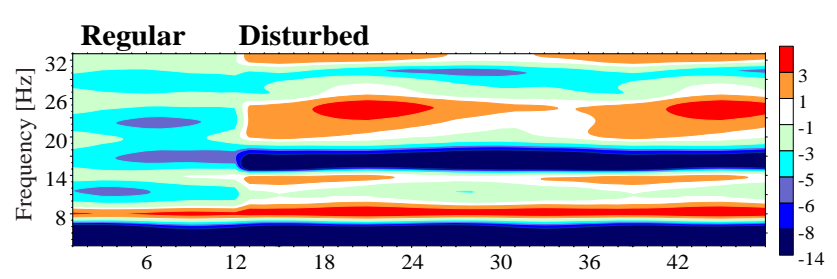

Fig. 14. Model dynamic spectrum of SR when a disturbance abruptly appears over Taiwan (at UT $=12 \mathrm{~h}$ )

clear that the implication of an increased $R_{a}$ factor simply raised the effect, so that a problem could happen with validity of the perturbation theory method employed in our computations. The results of computations are shown in Fig. 13. The left frame of Fig. 13 illustrates the frequency dependence of the dimensionless field disturbance, see Eq. (6), computed for the sources located in Asia, Africa, and America. One may see that the highest relative disturbance is connected to the Asian source and is observed around the $20-\mathrm{Hz}$ frequency (third SR mode). The plots in the left frame of Fig. 13 provide the "general picture" of the wave scattering, and one has to remember that the function $|\mathrm{B}(\mathrm{f})|$ may become large not only when the scattered field (denominator) is large, but also when the direct wave is small, e.g. at the nodal distance. This latter instance exactly the case for the source in Asia; see the lower frame in the right column of the plots in Fig. 13. The right plots in Fig. 13 show the spectra of the sole direct wave (no ionospheric disturbance) shown in a black line with corresponding spectra in the presence of the modification, given in a red line marked as "Disturbed". The lowest right frame depicts the data computed for the Asian source. One may see that the undisturbed field has a minimum around the $20-\mathrm{Hz}$ frequency. Therefore, the relevant peak appeared in the left frame for the Asian source. The spectra show that this source might produce a noticeable disturbance only around the second SR mode. The right-hand side plots of Fig. 13 indicate that only American thunderstorms are well positioned, causing a two-fold increase in the spectral amplitude at frequencies around $f_{4}$, as was already qualitatively described before. The non-uniformity over Taiwan cannot increase the amplitudes of the waves arriving from other global thunderstorm centers. As the possible conclusion, the presence of an ionospheric perturbation over Taiwan and the wave interference between the direct wave and that scattered from the ionospheric perturbation at Taiwan, act together as a frequency filter, which should be multiplied with the conventional SR frequency spectrum in which the intensity is decreasing with the increase in mode number. This hypothesis is likely to account for the major two points of our discovery (an enhancement at $\sim f_{4}$ and a possible shift in $f_{4}$ ). As seen in Fig. 13, the seismo-ionospheric perturbation is likely to influence the lower harmonic as well, but we will not discuss this in any detail.

As the global lightning activity drifts around the planet during the day, a question arises: "Why is the increase in the field amplitude observed all through the day?" The drifting activity must produce the effect varying in time. The controversy might be resolved in the following way. Long-term SR observations in the horizontal magnetic field indicate that the natural signal is a sum of two components: one of them is stable in time, forming a "podium" for the time-varying signal; see, e.g. SR records presented in Price and Melnikov (2004). We also know that there are two components; correspondingly the non-polarized and polarized signals (Nickolaenko et al., 2004). The time varying polarized part is associated with the electromagnetic radiation from the compact lightning activity. The stable podium or non-polarized component originates from the "background" thunderstorms that cover the whole globe: they occur everywhere and at any time. Pulsed signals from such strokes arrive at an observer along the azimuth, ranging from 0 to $2 \pi \pi$, and merge into a noncoherent depolarized noise. Thus, we may accept that any thunderstorm center "works" all through the day, with activity increasing by $30-50 \%$ during the local afternoon hours, so that the amplitude of SR never decreases substantially during the day.

The SR background signals permanently arrive at the Nakatsugawa observatory from all directions during the whole day, However, the "podium" signals coming from the American thunderstorms are reflected and enhanced by the ionospheric irregularity located over Taiwan. Thus, the field enhancement by the non-uniformity is observed all through the day in the vicinity of the fourth SR mode with minor amplitude variations.

Figure 14 demonstrates the model dynamic spectrum computed for two successive days. The time UT is shown on the abscissa in hours, the frequency is plotted on the ordinate in 
$\mathrm{Hz}$, and the logarithm of the field intensity is shown by inking. (do you mean shading?) The scale bar is given on the right side of the figure. Horizontal dashed grid lines indicate the usual positions of SR peaks in the spectrum. Vertical grid lines mark the 6-h intervals. The record of two days duration was modeled. There is no ionospheric perturbation during the first $12 \mathrm{~h}$ in the model dynamic spectrum, and regular diurnal variations are present there with SR peaks observed as a regular succession of horizontal lines. The form of spectrum abruptly changes after the ionosphere is modified over Taiwan (the choice of disturbance parameters is described below). The parts of the maps are marked as "regular" and "disturbed". As one may see, the general behavior found experimentally is pertinent to our model computations. The occurrence of Q bursts at $f_{4}$ is apparently due to the enhanced propagation characteristics at $\sim f_{4}$ because of the presence of seismo-ionospheric perturbations at Taiwan. The source is the same as the SR, but from a giant lightning in America.

We here comment on why no significant effect at Lekhta was observed. The geometry of the direct and reflected paths toward Lekhta indicates that there is no such lucky situation (as given in this paper): no backscatter. The theory (Nickolaenko and Hayakawa, 2002) indicates that at ELF only the overhead disturbance or a backscattering from a nearby disturbance may produce a noticeable effect. In this sense Lekhta is a remote place and we expect no backscattering signal.

Emergence of ionospheric perturbations prior to a large earthquake has already been evidenced by means of subionospheric VLF/LF propagation anomalies (Hayakawa et al., 1996; Molchanov et al., 1998). Because these subionospheric VLF/LF signals are known to be reflected from the lowest ionosphere (D region at day and lower E layer at night), it is already believed that the lowest ionosphere is perturbed a few days to about a week before large earthquakes. Our recent study with the use of over-horizon VHF signals has indicated the presence of atmospheric (altitude $\sim$ a few tens of $\mathrm{km}$ ) perturbations about one week before the earthquakes (Fukumoto et al., 2001). ELF propagation, including SR and ELF transients, is connected to two characteristic heights (h1 and h2) (Greifinger and Greifinger, 1978). The first altitude $\mathrm{h} 1$ is the height where the displacement current at a given frequency becomes equal to the conductivity current. It is the height at which the atmosphere becomes a conducting medium (the electric field does not penetrate to the altitudes above $\mathrm{h} 1$ ). The $\mathrm{h} 2$ is the height where the fields change from wave-like to diffusionlike (only the magnetic field component reaches the altitude $\mathrm{h} 2$ ). The characteristic value of h1 at SR frequencies is approximately $\mathrm{h} 1 \sim 50 \mathrm{~km}$, while $\mathrm{h} 2 \sim 90 \mathrm{~km}$ (Nickolaenko and Hayakawa, 2002; Mushtak and Williams, 2002). Therefore, we can understand that the height range responsible for ELF propagation is perturbed in association with earthquakes, taking into account the experimental or observational evidence of seismo-atmospheric and -ionospheric perturbations mentioned before. The scale of the perturbation in the upper ionosphere is estimated as radius $\mathrm{R}($ in $\mathrm{km})=\exp (\mathrm{M})$, where
M is the magnitude (Ruzhin and Depeuva, 1996). This yields $\mathrm{R} \sim 2000 \mathrm{~km}$ for the magnitude of the Chi-chi earthquake $(\mathrm{M}=7.6)$, which means that seismo-atmospheric and seismoionospheric perturbations have the spatial scale of, at least, one $\mathrm{Mm}$. These perturbations seem to be ready to reflect the ELF waves just around the epicenter of the Chi-chi earthquake (in Taiwan) to be observed at the observatory. Liu et al. (2000) have studied the $f_{0} \mathrm{~F} 2$ for this Chi-chi earthquake, and have found that the precursors appeared 1-6 days prior to this earthquake in the form of the recorded $f_{0} \mathrm{~F} 2$ decrease below its associated lower bound. This means that some kind of anomaly is surely occurring in the ionosphere. Of course, the generation mechanism of seismo-ionospheric perturbations is not well established, but Hayakawa et al. (2004) have suggested a few possible mechanisms.

Lastly, we comment on the geomagnetic activity during the relevant period, because it may be possible that the geomagnetic activity may influence the SR anomaly. Though not shown as a figure, the geomagnetic activity is not severely disturbed, so that the present SR anomaly is likely to have nothing to do with the geomagnetic activity.

Finally, we can suggest the lithospheric source. Kulchitsky et al. (2004) have performed the detailed computations of ULF/ELF waves in the complicated lithospheric structure. We have to try to find any resonance effects in the lithospheric waveguide (or cavity).

Acknowledgements. This study is partly supported by the Mitsubishi Foundation and Japan Society of Promotion of Science (\#15403012), to which we are grateful.

Topical Editor T. Pulkkinen thanks K. Hattori and another referee for their help in evaluating this paper.

\section{References}

Fukumoto, Y., Hayakawa, M., and Yasuda, H.: Investigation of over-horizon VHF radio signals associated with earthquakes, Natural Hazards Earth System Sci., 1, 127-136, 2001.

Greifinger, C. and Greifinger, P.: Approximate method for determining ELF eigen-values in the Earth-ionosphere waveguide, Radio Sci., 13, 831-837, 1978.

Hattori, K.: ULF geomagnetic changes associated with large earthquake, Terr. Atmos. Ocean. Sci., 15, 329-360, 2004.

Hayakawa, M. and Fujinawa, Y.: Electromagnetic Phenomena Related to Earthquake Prediction, Terra Sci. Pub. Co., Tokyo, 667, 1994.

Hayakawa, M.: Whistlers, in "Handbook of Atmospheric Electrodynamics”, Volland, H.(Ed.), 1, 155-193, CRC Press, Boca Raton, 1995.

Hayakawa, M., Molchanov, O. A., Ondoh, T., and Kawai, E.: The precursory signature effect of the Kobe earthquake on VLF subionospheric signals, J. Comm. Res. Lab., Tokyo, 43, 169180, 1996.

Hayakawa, M.: "Atmospheric and Ionospheric Electromagnetic Phenomena Associated with Earthquakes", TERAPPUB, Tokyo, 966, 1999.

Hayakawa, M. and Molchanov, O. A.: "Seismo Electromagnetics: Lithosphere- Atmosphere - Ionosphere Coupling", TERRAPUB, Tokyo, 477, 2002. 
Hayakawa, M., Hattori, K., and Ando, Y.: Natural electromagnetic phenomena and electromagnetic theory, Inst. Electr. Engs. Japan (IEEJ), Trans. Fundamentals and Materials, 124, 1, 72-79, 2004.

Hayakawa, M.: Is earthquake prediction possible by means of electromagnetic phenomena associated with earthquakes?, IEEJ, Trans. Fundamentals and Materials, 124, 1, 3-4, 2004.

Hayakawa, M. and Hattori, K.: Ultra-low-frequency electromagnetic emissions associated with earthquakes (Invited paper), in "Special Issue on Recent Progress in Electromagnetic Theory and Its Application", Inst. Electr. Engrs. Japan, Trans. Fundamentals and Materials, 124, 1101-1108, 2004.

Hayakawa, M., Molchanov, O. A., and NASDA/UEC team: Summary report of NASDA's earthquake remote sensing frontier project, Special Issue on "Seismo Electromagnetics and Related Phenomena”, Hayakawa, M., Molchanov, O. A., Biagi, P., and Vallianatos, F. (Eds.), Phys. Chem. Earth, 29, 617-626, 2004.

Kulchitsky, A., Ando, Y., and Hayakawa, M.: Numerical analysis on the propagation of ULF/ELF signals in the lithosphere with highly conductive layers, Phys. Chem. Earth, 29, 551-557, 2004.

Liu, J. Y., Chen, Y. I., Pulinets, S. A., Tsai, Y. B., and Chuo, Y. J.: Seismo-ionospheric signatures prior to $\mathrm{M} \geq 6.0$ Taiwan earthquakes, Geophys. Res. Lett., 27, 3113-3116, 2000.

Molchanov, O. A., Hayakawa, M., Ondoh, T., and Kawai, E.: Precursory effects in the subionospheric VLF signals for the Kobe earthquake, Phys. Earth Planet. Inter., 105, 239-248, 1998.

Molchanov, O. A. and Hayakawa, M.: Subionospheric VLF signal perturbations, possibly related to earthquakes, J. Geophys. Res., 103, 17 489-17 504, 1998.

Mushtak, V. C. and Williams, E. R.: ELF propagation parameters for uniform models of the Earth-ionosphere waveguide, J. Atmos. Solar-terr. Phys., 64, 1989-2001, 2002.
Nickolaenko, A. P.: ELF radio wave propagation in a locally nonuniform Earth-ionosphere cavity, Radio Sci., 29, 1187-1199, 1994.

Nickolaneko, A. P. and Hayakawa, M.: "Resonances in the Earthionosphere Cavity”, Kluwer Acad. Pub., Dordrecht, 380, 2002.

Nickolaenko, A. P., Rabinowicz, L. M., Shvets, A. V., and Schekotov, A. Yu.: Polarisation characteristics of low frequency resonances, Izvestija VUZov, Radiofizika, XLVII, (in Russian), 267291, 2004.

Ogawa, T., Tanaka, Y., Fraser-Smith, A. C., and Gendrin, R.: Worldwide simultaneity of a Q-burst in the Schumann resonance frequency range, J. Geomagn. Geolectr., 19, 377-384, 1967.

Ohta, K., Umeda, K., Watanabe, N., and Hayakawa, M.: ULF/ELF emissions observed in Japan, possibly associated with the Chichi earthquake, Natural Hazards Earth System Sci., 1, 37-42, 2001.

Pappert, R. A.: Effect of a large patch of sporadic E on night-time propagation at lower ELF, J. Atmos. Terr. Phys., 42, 417-425, 1981.

Price, C. and Melnikov, A.: Diurnal, seasonal and inter-annual variations in the Schumann resonance parameters, J. Atmos. Solarterr. Phys., 66, 1179-1185, 2004.

Ruzhin, Yu. Ya. and Depueva, A. Kh.: Seismoprecursors in space as plasma and wave anomalies, J.Atmos. Electr., 16, 3, 271-288, 1996.

Sentman, D.: Schumann resonances, in "Handbook of Atmospheric Electrodynamics”, Volland, H. (Ed.), 1, 267-310, CRC Press, Boca Raton, 1995. 